Journal of Complex Networks (2021) Page 1 of 17

doi:10.1093/comnet/xxx000

\title{
An Adaptive Bounded-Confidence Model of Opinion Dynamics on Networks
}

\author{
UNCHITTA KAN \\ Department of Computational and Data Sciences, George Mason University \\ MiCHELle FENG \\ Department of Computing + Mathematical Sciences, California Institute of Technology \\ AND \\ MASON A. PORTER \\ Department of Mathematics, University of California, Los Angeles and Santa Fe Institute
}

[Received on 10 December 2021]

\begin{abstract}
Individuals who interact with each other in social networks often exchange ideas and influence each other's opinions. A popular approach to studying the dynamics of opinion spread on networks is by examining bounded-confidence (BC) models, in which the nodes of a network have continuous-valued states that encode their opinions and are receptive to other opinions if they lie within some confidence bound of their own opinion. We extend the Deffuant-Weisbuch (DW) model, which is a well-known BC model, by studying opinion dynamics that coevolve with network structure. We propose an adaptive variant of the DW model in which the nodes of a network can (1) alter their opinion when they interact with a neighboring node and (2) break a connection with a neighbor based on an opinion tolerance threshold and then form a new connection to a node following the principle of homophily. This opinion tolerance threshold acts as a threshold to determine if the opinions of adjacent nodes are sufficiently different to be viewed as discordant. We find that our adaptive $\mathrm{BC}$ model requires a larger confidence bound than the standard DW model for the nodes of a network to achieve a consensus. Interestingly, our model includes regions with 'pseudo-consensus' steady states, in which there exist two subclusters within an opinion-consensus group that deviate from each other by a small amount. We conduct extensive numerical simulations of our adaptive BC model and examine the importance of early-time dynamics and nodes with initial moderate opinions for achieving consensus. We also examine the effects of coevolution on the convergence time of the dynamics.
\end{abstract}

Keywords: opinion dynamics, bounded-confidence models, coevolving networks, homophily

2020 Math Subject Classification: 91D30, 05C82, 91C99

\section{Introduction}

When individuals in a social network interact with each other, they can discuss and exchange ideas, and they can thereby influence each other's opinions. In social networks, similar individuals are more likely than dissimilar individuals to engage with each other [23]. Such homophilic behavior can lead to homophilic communities and the formation of 'echo chambers' on social and political issues [8, 14]. Homophily is thus a key lens to use when studying the formation and spread of opinions on social networks [28, 31]. See [13] for a review of homophily in social network analysis.

Researchers have developed many models of opinion dynamics [5, 17, 27], and network structure can exert a significant influence on opinion dynamics on networks $[4,30]$. In a model of opinion 
dynamics, the opinions of the nodes (which represent agents, such as individual humans or other entities) can take either discrete values (such as in a classical voter model [12], in which there are two possible opinion states) or continuous values. Well-known examples of the latter are bounded-confidence models [27], in which opinions take values either in an interval or in a higher-dimensional space. Allowing the opinion states of nodes to be in an interval is useful for modeling opinions on a liberal-conservative political spectrum or on a single issue, and similar interpretations are possible when one considers two or more opinion dimensions [3].

In a bounded-confidence (BC) model, when two agents interact, they update their opinions by compromising by some amount if the difference between their opinions is below a specified threshold (the confidence bound). Otherwise, following standard practice, we suppose the two agents do not adjust their opinions when they interact with each other. ${ }^{1}$ One way to interpret an agent's confidence bound is as its willingness to engage with people who have different ideologies. (For example, perhaps it encodes their open-mindedness.) The two most famous BC models are the Deffuant-Weisbuch (DW) model [6] and the Hegselmann-Krause (HK) model [10], which were both introduced about two decades ago. In the DW model, which we will generalize in the present paper, one considers asynchronous updates of node opinions and randomly chooses a single node pair (i.e. dyad) to interact in each time step. One then applies the opinion-update rule that we described above. In the HK model, node opinions update synchronously.

Since the pioneering works of Deffuant et al. [6] and Hegselmann and Krause [10], there have been many studies of $\mathrm{BC}$ models (see, e.g. [20, 21, 25, 31, 34]), typically in the form of numerical investigations. $\mathrm{BC}$ models have been extended in a variety of ways, such as by incorporating content sharing and media nodes [3], updating opinions based on the median opinion (instead of the mean opinion) of interacting agents [24], and considering polyadic interactions of agents instead of only dyadic ones [11].

In the present paper, we generalize the DW model by constructing an adaptive network model in which BC opinion dynamics coevolve with network structure. Although there is a wealth of research on opinion models in adaptive networks [9, 32], especially in the form of adaptive voter models (see, e.g. $[7,22,33])$, there have only been a few studies of adaptive BC models [2, 15, 16, 29, 31]. Kozma and Barrat examined DW dynamics on adaptive Erdôs-Rényi (ER) network. They modeled the coevolving network and opinion dynamics using a biased coin flip. One chooses a dyad uniformly at random. With probability $p$, if the difference in the opinions of the two nodes in the dyad is larger than a confidence bound, the edge that is attached to one of those nodes rewires to some other node that one chooses uniformly at random. With probability $1-p$, the node opinions evolve according to the DW opinionupdate rule. More recently, Parravano et al. [29] studied a coevolving BC with signed edges (because nodes can be friends or enemies), with sign changes that depend on the distances between node opinions; Brede [2] used an adaptive BC model to study the active participation of agents (who deliberately seek out other agents to try to change the opinions of those agents) in consensus formation; and Sasahara et al. [31] adapted a BC model to model opinion dynamics in the context of online social platforms in which users can encounter messages, repost messages, and unfollow users.

We develop an adaptive DW model that incorporates an opinion tolerance threshold and a rewiring mechanism that follows the principle of homophily. In each time step of our model, we update the opinions of nodes using the usual BC mechanism and we break the edges between probabilistically selected neighboring nodes whose opinions are too far apart (i.e. they exceed the opinion tolerance

\footnotetext{
${ }^{1}$ An alternative choice is for such agents to "dig in their heels" and adjust their opinions so that they are farther apart from each other.
} 
threshold and are thus 'discordant'). When an edge breaks, one of these nodes connects to a new node with a probability that depends on the similarity of their opinions (i.e. based on homophily). Our adaptive BC model requires a larger confidence bound than the standard DW model for the nodes of a network to achieve a consensus. In some regions of parameter space, we observe the emergence of a 'pseudo-consensus', in which there exist two subclusters within an opinion-consensus group that deviate from each other by a small amount. When the agents have a low tolerance for neighbors with different opinions from theirs, our model behaves differently from the standard DW model. When the confidence bound is small, our model leads to faster cluster formation (and hence to a faster convergence time) than in the standard DW model. When the confidence bound is large, our model has long convergence times, which is the opposite of the standard DW model.

Our study has some similarities to $[15,16]$, but a crucial difference is that our rewiring rule is based on the sociological principle of homophily [13]. The connections that people form on social media [1] (and elsewhere) are influence heavily by homophily, and it is important to incorporate such ideas into models of coevolving networks. In our model, agents both compromise their opinions with like-minded individuals (according to a $\mathrm{BC}$ mechanism) and dissolve old connections and form new connections based on the similarities in their opinions. ${ }^{2}$ There are two key differences between our adaptive BC model and existing adaptive BC models. First, each agent in our model has not only a confidence bound but also a rewiring threshold (an 'opinion tolerance threshold') that determines the amount of opinion difference that it can tolerate. This yields a notion of discordant edges, which is an idea that has been important in the study of adaptive voter models [7]. Second, new connections form between two given nodes with a larger probability when they have similar opinions, instead of nodes rewiring to a new node uniformly at random. This resembles studies in adaptive voter models that have compared the effects of rewiring to nodes with exactly the same opinion versus rewiring to nodes regardless of their opinion [7]. In the context of our adaptive BC model, perhaps individuals with a high opinion tolerance threshold are willing to interact with individuals whose opinions are very different from theirs, even though their own opinion will not be influenced by theirs unless their opinions are also within the confidence bound. There are also recent studies of adaptive opinion models with homophilic rewiring that use frameworks for opinion dynamics that are different from BC models $[18,19]$.

Our paper proceeds as follows. In Section 2, we give background information on the DW model. In Section 3, we present our adaptive BC model and discuss some notes about our implementation of it. In Section 4, we discuss the results of numerical computations of our model. We conclude in Section 5. Our code is available at https://gitlab.com/unchitta/coevolving-bc.

\section{The Deffuant-Weisbuch Model}

We briefly review the Deffuant-Weisbuch (DW) model of BC opinion dynamics [6]. Consider a network of agents in which each agent $i$ holds an opinion $x_{i}$ that changes with time. In the original DW model, the agents mix completely, so BC dynamics occur on a complete graph. In a given time step (which has a small size $\Delta t$ ), we choose two agents, $i$ and $j$, uniformly at random to interact with each other. If their opinions, $x_{i}(t)$ and $x_{j}(t)$, at time $t$ satisfy $\left|x_{i}(t)-x_{j}(t)\right|<C$ for some confidence bound $C$ (which one can interpret as the open-mindedness of the agents), they compromise their opinions through the

\footnotetext{
${ }^{2}$ It would also be interesting to consider attributes (such as similar demographic characteristics or hobbies) other than opinions.
} 
following opinion-update rule:

$$
\begin{aligned}
& x_{i}(t+\Delta t)=x_{i}(t)+\alpha\left(x_{j}(t)-x_{i}(t)\right), \\
& x_{j}(t+\Delta t)=x_{j}(t)+\alpha\left(x_{i}(t)-x_{j}(t)\right),
\end{aligned}
$$

where $\alpha \in(0,0.5]$ is a constant that is often called a 'convergence parameter' because it affects the speed of convergence. The parameter $\alpha$ governs how much agents compromise when they update their opinions. In the present paper, we use a time interval of $\Delta t=1$.

As is typical in studies of BC models [25], we assume that all agents have the same confidence bound. In this scenario, when the dynamics reach a steady state, researchers have observed the formation of consensus and fragmented clusters of opinions, depending on the value of the confidence bound. Situations with exactly 2 clusters are 'polarized' and situations with 3 or more clusters are 'fragmented'. As one increases the confidence bound, there is a phase transition from polarized/fragmented states to consensus states [20]. When agents are more receptive to different opinions (i.e. when their confidence bound is larger), they are more likely to compromise with more of the other agents. In the adaptive DW model in $[15,16]$, the critical point of this phase transition occurs at a larger confidence bound than in the standard DW model. In the polarized and fragmented regimes, which occur when the confidence bound is small, there are peaks (which indicate the presence of multiple opinion clusters) in the steadystate distribution of opinions; additionally, the number of peaks and the distance between the opinions in the clusters depend on the confidence bound [6]. The number of agents in a system affects the time to converge to a steady state, but it typically does not affect the steady state itself.

\section{Our Adaptive BC Model}

Consider an undirected network $G=(V, E)$ (i.e. graph), where $V$ is the set of nodes in the network and $E$ is the set of edges between them. The network coevolves with the opinions of the nodes, so the set of edges can change with time and it is helpful to write $E=E(t)$. The node set $V$ is constant in time, and $N=|V|$ is the number of nodes in the network. Let $C \in[0,1]$ be the confidence bound, and let $x_{i}(t) \in[0,1]$ denote node $i$ 's opinion about some issue. The set of discordant edges is $E_{d}^{\beta}(t)=\{(i, j) \in$ $\left.E(t):\left|x_{i}(t)-x_{j}(t)\right|>\beta\right\}$. That is, $E_{d}^{\beta}(t)$ is the set of dyads in the graph $G=G(t)$ whose opinions are farther apart than the opinion tolerance threshold $\beta$ at time $t$. We view the threshold $\beta$ as a homophilic parameter.

The time $t$ is discrete, and two processes occur in series in each time step. In the first process, nodes rewire; in the second process, nodes update their opinions. In the rewiring process, we choose up to $M$ discordant edges to rewire according to a homophilic rewiring rule that typically changes the network structure. We describe this mechanism in Section 3.1. We then choose $K$ dyads; the nodes in the dyad adjust their opinions according to the update rule (2.1) of the DW model [6]. We give more details in Section 3.2.

In a real-world context, the first process may correspond to an individual 'unfriending' one of their connections on a social network (e.g. on social media) and establishing a friendship with someone else instead. We assume that this occurs when the individual cannot tolerate the difference between their opinion and that of the node that it is unfriending. The individual then chooses to befriend someone else. Based on the principle of homophily, the individual is more likely to befriend somebody with an opinion that is similar to theirs than somebody whose opinion is very different from theirs. We illustrate this process in Figure 1. After this rewiring process, we choose dyads for opinion updating; the nodes in these independently-chosen dyads update their opinions in the same way as in the DW model. 
Schematic illustration of rewiring procedure: $C=0.3 ; \beta=0.2$
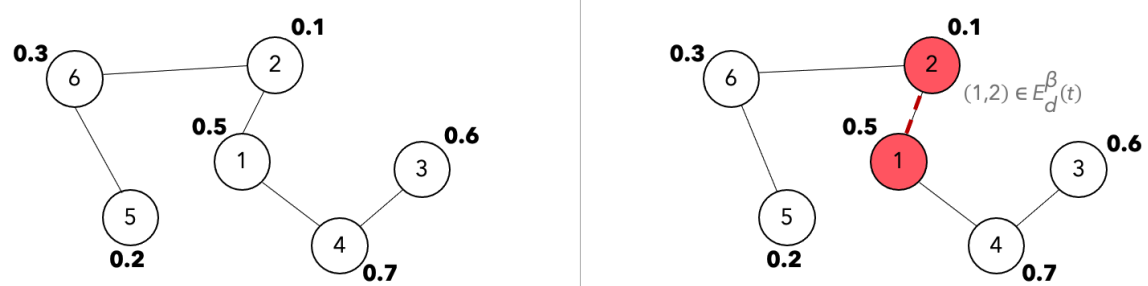

(a) (b)

(c) (d)
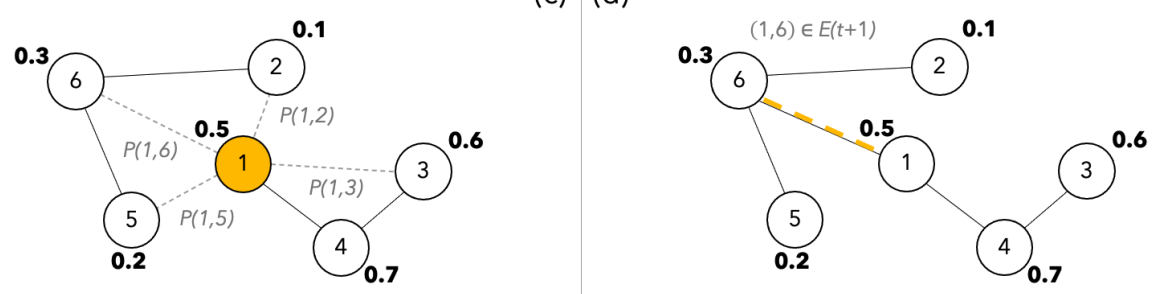

$P(1,3)>P(1,6)>P(1,5)>P(1,2)$

FIG. 1: A schematic illustration of our adaptive BC model's rewiring mechanism. In this example, the confidence bound is $C=0.3$ and the opinion tolerance threshold is $\beta=0.2$. We show the current network structure and node opinions in (a). In the depicted time step, we choose the edge $(1,2)$ uniformly at random from the set of discordant edges and remove this edge (see (b)). As we illustrate in (c), we then choose node 1 to rewire to a new node. (We select which of nodes 1 or 2 rewires with equal probability.) The rewiring probabilities depend on each node's opinions in this time step. In this example, we form the new edge $(1,6)$ (see $(d))$.

The two processes occur until there are no further noticeable changes of the opinions of the nodes in the network. In our computational experiments, we stop a simulation if the sum of the changes to node opinions is less than $10^{-5}$ for each of 100 consecutive time steps. This is our numerical tolerance for convergence. Because it is possible that the simulations may fail to satisfy this termination criterion, we set a 'bail-out time' and stop a simulation after $10^{6}$ time steps if it has not already stopped.

We give the pseudocode for our adaptive BC model in Algorithm 1. Our code is available at https : //gitlab.com/unchitta/coevolving-bc.

\subsection{Homophilic Rewiring}

In our model's rewiring process, we select $M$ edges uniformly at random from the set $E_{d}^{\beta}(t)$ of discordant edges. If $\left|E_{d}^{\beta}(t)\right|<M$, we select all of the discordant edges. We remove each edge $(i, j)$ in this set, and we select node $i$ or $j$ with equal probability to form an edge to a new node $a$. We choose the node $a$ with a probability that depends on the similarity between its opinion and the selected node's opinion. To work with mathematically well-defined similarities, we consider similarities of opinions and define a metric $d(x, y)$ on the space of node opinions. One can also define similarities in higher 


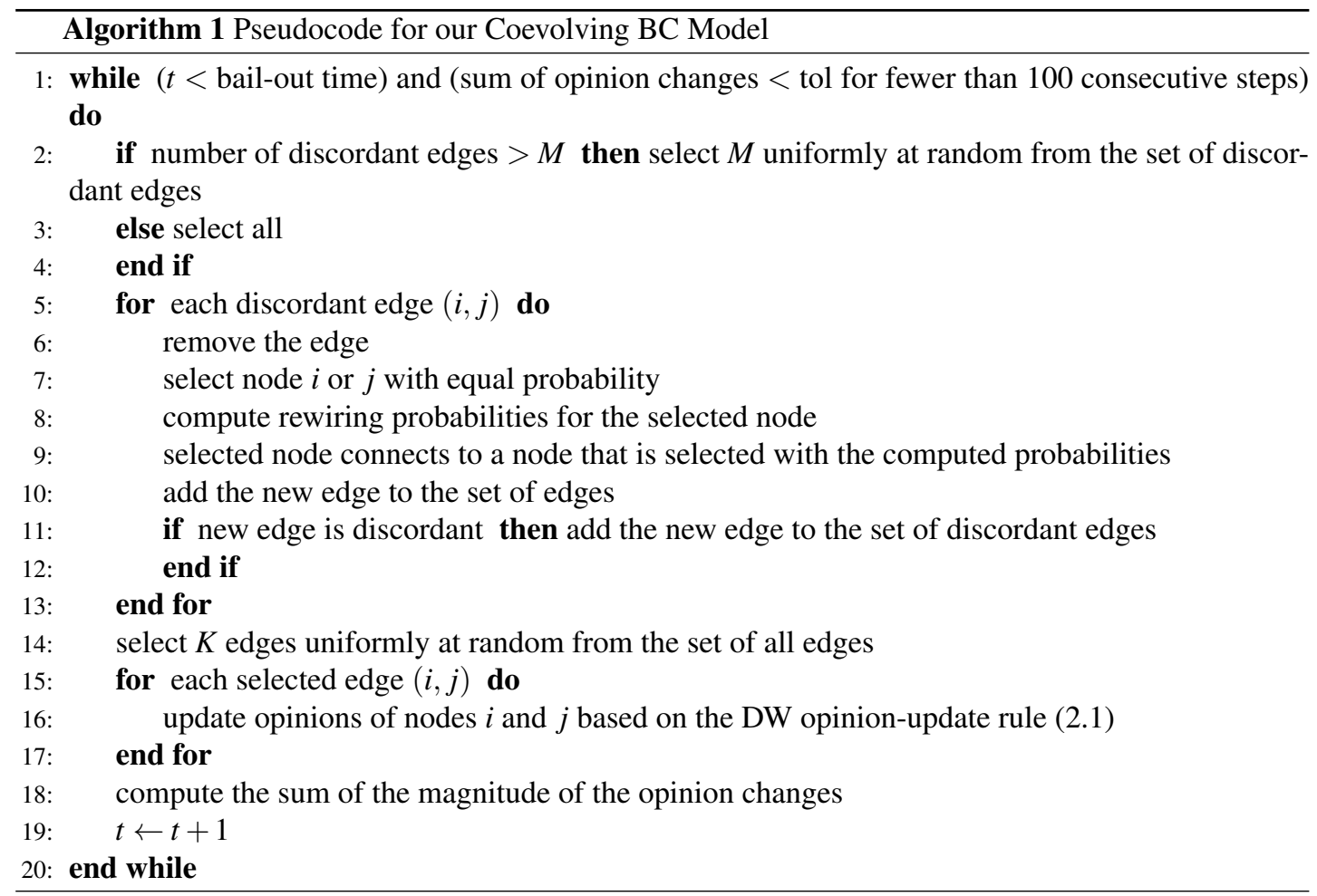

dimensions either by considering higher-dimensional opinion spaces or by defining similarities that also incorporate node attributes other than their current opinion (see, e.g., [3]).

Suppose that we choose agent $i$ from the dyad $(i, j)$. The probability that agent $i$ rewires to some other agent $a$ is

$$
\mathbb{P}(i \rightarrow a)=\frac{1}{D}\left(1-d\left(x_{i}, x_{a}\right)\right),
$$

where the proportionality constant is the normalization constant (which we will specify shortly) and $d(x, y)$ is a metric (e.g. the $L_{1}$ norm or the $L_{2}$ norm). In the present paper, we use the $L_{2}$ norm, so $d=\|\cdot\|_{2}$. We set $\mathbb{P}(i \rightarrow i)$ to 0 to prevent self-edges, and we set the probability that $i$ rewires to one of its existing connections to 0 to avoid multi-edges. We include constant $D$ is in Eq. (3.1) because we need a normalization to guarantee that we have a probability. We calculate $D$ by solving $\sum_{z} \mathbb{P}(i \rightarrow z)=\frac{1}{D} \sum_{z}\left[1-d\left(x_{i}, x_{z}\right)\right]=1$.

After choosing an agent $a$ randomly according to the probabilities in (3.1), we add the new edge $(i, a)$ to $E(t+1)$. If the new edge is discordant, we also add it to $E_{d}^{\beta}(t)$. We allow $i$ to rewire to $j$, even though the former just unfriended the latter. Sometimes, life just works that way (and it is convenient for our computations).

In Figure 1, we illustrate the rewiring process with example values of the confidence bound $C$ and the opinion tolerance threshold $\beta$. 


\subsection{Opinion Updates}

After the rewiring step, we select $K$ dyads uniformly at random, and we adjust the opinions of the nodes in the dyad using the DW opinion-update rule (2.1). Specifically, at time step $t$, two interacting agents $i$ and $j$ adjust their opinions if the difference between their opinions is less than or equal to the confidence bound (i.e. if $\left.\left|x_{i}(t)-x_{j}(t)\right| \leqslant C\right)$. They change their opinions according to the update rule

$$
\begin{aligned}
& x_{i}(t+1)=x_{i}(t)+\alpha\left(x_{j}(t)-x_{i}(t)\right), \\
& x_{j}(t+1)=x_{j}(t)+\alpha\left(x_{i}(t)-x_{j}(t)\right),
\end{aligned}
$$

where $\alpha$ is the convergence parameter. If the opinions of $i$ and $j$ are not within the confidence bound, they do not change their opinions. The parameters $M$ (i.e. the number of discordant edges that we rewire in one time step) and $K$ also affect the rate of convergence to a steady state. For example, a larger value of $K$ signifies that there are more encounters between agents in each time step, so more agents can compromise their opinions in a single time step.

It is possible that we select the same node $h$ in more than 1 of the $K$ dyads in a time step. In this case, we apply the opinion update (3.2) each time that we select $h$.

We run our simulations - with first the rewiring process and then the opinion-update process at each time step - until the system converges to a steady state (within the numerical tolerance level that we discussed previously) or until we reach the bail-out time. The steady state can include one or more opinion clusters. The quantity $M / K$ determines the relative time scales of the rewiring dynamics and the opinion dynamics. When $M / K \ll 1$, the opinions of the nodes change much faster than the network structure; by contrast, when $M / K \gg 1$, network structure changes much faster than the opinions.

\section{Numerical Simulations}

Because we need to consider both changes in opinions and changes in network structure, it is natural to ask how these changes affect each other. We explore the interaction between these two aspects of our model's dynamics by examining both network topology and the opinion states as a function of time. We present numerical results from running simulations on synthetic networks that we generate using the $G(N, p)$ ER random-graph model [26]. It is useful to recall the parameters for our adaptive BC model: the number $N$ of nodes in a network, the maximum number $M$ of edges that we rewire in one time step, the number $K$ of dyads that we consider when updating opinions, the convergence parameter $\alpha$ of the DW opinion-update rule (3.2), the confidence bound $C$, and the opinion tolerance threshold $\beta$. For a network that we construct using the $G(N, p)$ model, the connection probability between nodes is $p=\langle k\rangle / N$, where $\langle k\rangle$ is the mean degree of the network. We set $N=1000,\langle k\rangle=10, M / K=1 / 5$, and $\alpha=0.1$. We seed the initial opinion profile (i.e. the set of opinions of the agents) to be $X \in[0,1]^{N}$ randomly from the uniform distribution Unif $[0,1]$. In Figure 2, we show the results of a single trial of our model with $C=0.24$ and $\beta=0.32$.

Our code, along with the seed number that we use for our simulations, is available at https: //gitlab.com/unchitta/coevolving-bc.

\subsection{Concepts, Definitions, and Other Specifications}

Before presenting our results, we outline several concepts are helpful for understanding the behavior of our model. In the standard DW model, agents are in consensus when they have the same opinion. 

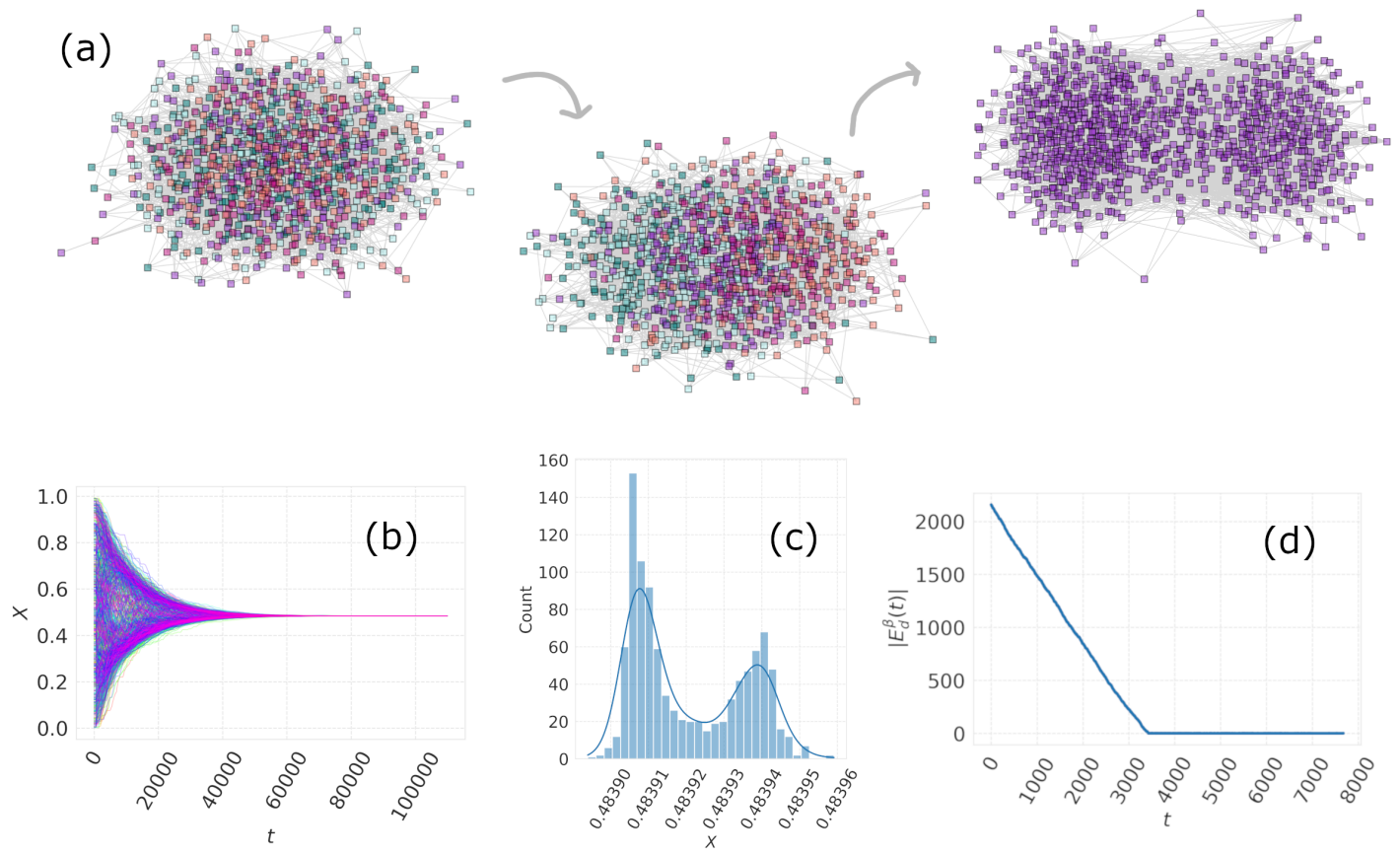

FIG. 2: Overview of one trial of our adaptive BC model with a confidence bound of $C=0.24$ and an opinion tolerance threshold of $\beta=0.32$. We show (a) the changes in the network structure with time, (b) the time evolution of the opinions of the agents in the network, (c) the distribution of the opinions of the agents at steady state, and (d) the number of discordant edges as a function of time. The colors in (a) and (b) indicate opinion values.

Additionally, a state with 2 clusters of opinions is polarized and a state with at least 3 clusters of opinions is fragmented. In our model, it is convenient to relax these notions a bit. For example, our notion of consensus includes situations in which nodes are almost (but not perfectly) in agreement.

Transition between polarization/fragmentation and consensus. In the standard DW model, when the confidence bound $C$ is small and below some critical value $C^{\prime}$, the steady state has multiple clusters of opinions (in different areas of the space of opinions). Intuitively, when $C$ is small, the agents in a network are close-minded and only interact with like-minded individuals, so opinion clusters emerge. Typically, we obtain more opinion clusters for progressively more close-minded agents. As agents become more open-minded (i.e. for larger values of $C$ ), more individuals engage with each other, which leads to more compromises and less fragmentation into different opinion clusters. If agents are sufficiently openminded (specifically, if $C<C^{\prime}$ ), they can reach a consensus, so our model appears to have a phase transition at the critical value $C^{\prime}$. Because $C^{\prime}$ can depend on $\beta$, we denote putative transition values in our model by $C_{\beta}^{\prime}$

Polarization and fragmentation. When consensus does not occur, we can further characterize the opinion clusters. We use the term 'major cluster' for any opinion clusters with at least at least 50 nodes (i.e. at least $5 \%$ of the $N=1000$ nodes in the network) and 'minor cluster' for any opinion cluster with 
(a)

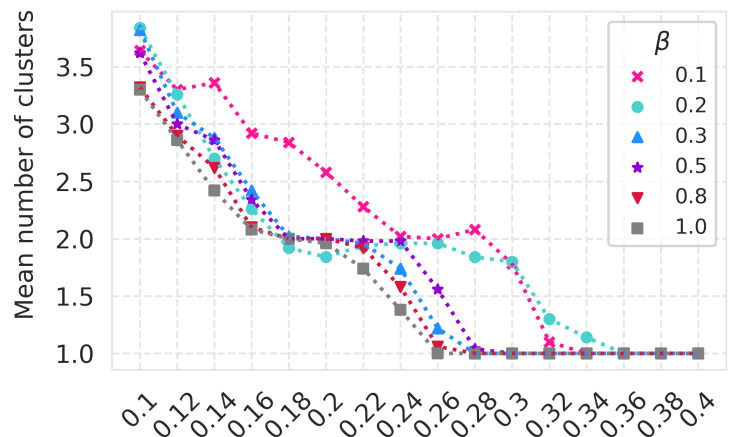

(b)

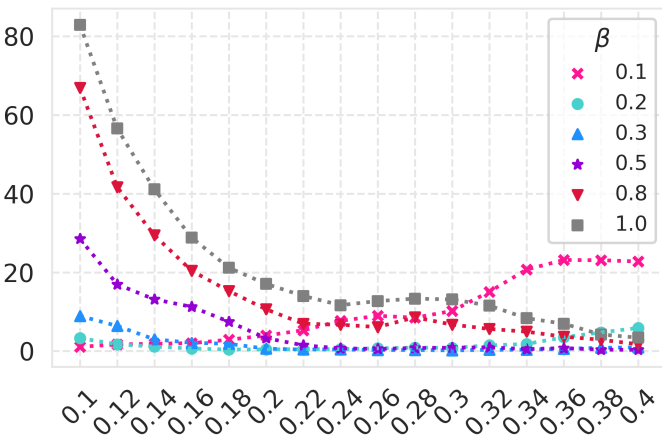

Confidence bound $(C)$

FIG. 3: The number of (a) major opinion clusters and (b) minor opinion clusters in our adaptive BC model at steady state as a function of the confidence bound $C$ for different values of the opinion tolerance threshold $\beta$. The results that we show are means of 50 trials. We initialize each trial with an independently generated ER graph and a different opinion profile that we draw from the uniform distribution Unif $[0,1]$.

fewer than 50 nodes. We use the term 'polarization' for situations with exactly 2 major clusters at steady state and 'fragmentation' for situations with 3 or more major clusters at steady state.

Consensus and pseudo-consensus. In a consensus regime, there is only 1 major cluster, with either a single consensus opinion or with opinions that are almost in perfect consensus. In particular, we observe situations in which (upon closer inspection) a major cluster has subclusters with opinions that differ from each other by a small value $\varepsilon$. In our model, this situation has an associated community structure. We refer to this type of consensus as a 'pseudo-consensus'.

Throughout our discussion, we often refer to the values of the opinion tolerance threshold $\beta$ and the confidence bound $C$ as being 'small', 'intermediate', or 'large'. For small $\beta$, agents are more aggressive at cutting ties with other agents with differing views and then befriending agents with similar opinions. For small $C$, agents are close-minded and are influenced only by agents with similar opinions as theirs. These two key parameters affect the time scales of the rewiring, the opinion updates, and their relation to each other. As we will see in our numerical experiments, different combinations of $\beta$ and $C$ can lead to very different behaviors at steady state. In practice, we observe that agents cut ties frequently for $\beta \lesssim 0.2$, so we refer to such values of $\beta$ as 'small'. Analogously, we observe that agents are closeminded for $C \lesssim 0.2$, so we refer to such values of $C$ as 'small'. Additionally, $\beta$ is 'intermediate' when $0.2 \lesssim \beta \lesssim 0.4$ and $C$ is 'intermediate' when $0.2 \lesssim C \lesssim 0.3$. Finally, $\beta$ is 'large' when $\beta \gtrsim 0.4$ and $C$ is 'large' when $C \gtrsim 0.3$. 


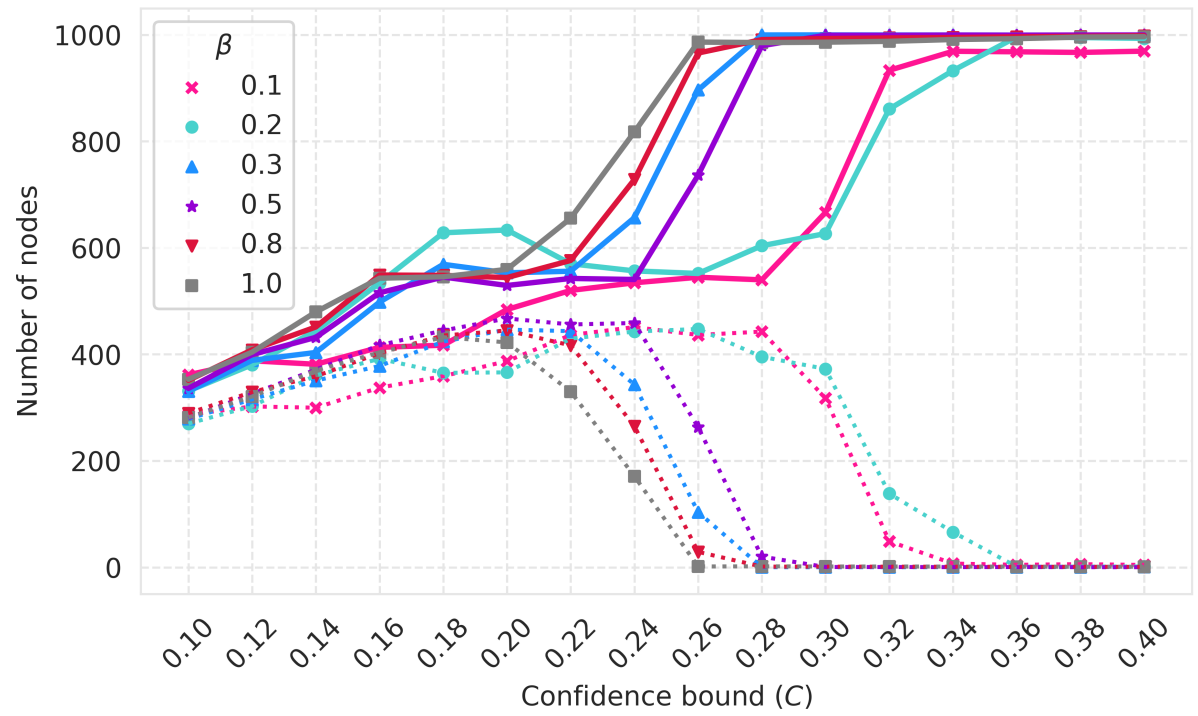

FIG. 4: The numbers of agents in the largest opinion cluster (solid curves) and the second-largest opinion cluster (dotted curves) versus the confidence bound $C$ for different values of the opinion tolerance threshold $\beta$. The results that we show are means of the same 50 trials as in Figure 3.

\subsection{Baseline Case (i.e. $\beta=1$ )}

When $\beta=1$ in our adaptive DW model, there is no rewiring because the set of discordant edges is always empty, so our model reduces to a standard variant of the standard DW. Therefore, we treat the results that we obtain with $\beta=1$ as a baseline. The baseline model should behave like the standard DW model, but with faster convergence times because more nodes change opinions in a single time step.

As we mentioned earlier, the standard (i.e. non-coevolving) DW model appears to have a phase transition between consensus and polarized/fragmented steady states at a critical value of the confidence bound. For the standard DW model on a complete network, this critical value appears to be $C^{\prime} \approx 0.26$ based on the numerical simulations in $[6,20]$. The numerical simulations of the DW model in [15] yield $C^{\prime} \approx 0.256$. In our model, $C^{\prime}$ can depend on $\beta$, so we denote putative transition values by $C_{\beta}^{\prime}$. In our baseline model (i.e. when $\beta=1$ ), we observe a transition between consensus and polarization at $C_{\beta=1}^{\prime} \approx 0.26$. This is consistent with the results in $[6,15,20]$.

In Figure 3(a) (where the gray curve corresponds to the baseline case), we show the number of major opinion clusters at steady state. The opinion profile fragments into 2-4 major clusters when $C \in[0.1,0.16)$ and is polarized when $C \in[0.16,0.26)$. When $C \gtrsim 0.26$, we always reach a consensus, except for very small ('minor') opinion clusters (some of which consist of isolated nodes). In Figure 4 , we show the number of nodes in the largest opinion cluster at steady state; in the polarized regime, the two largest opinion clusters each have almost 500 nodes. In Figure 5, we show the mean opinion of each major cluster as a function of the confidence bound $C$. 

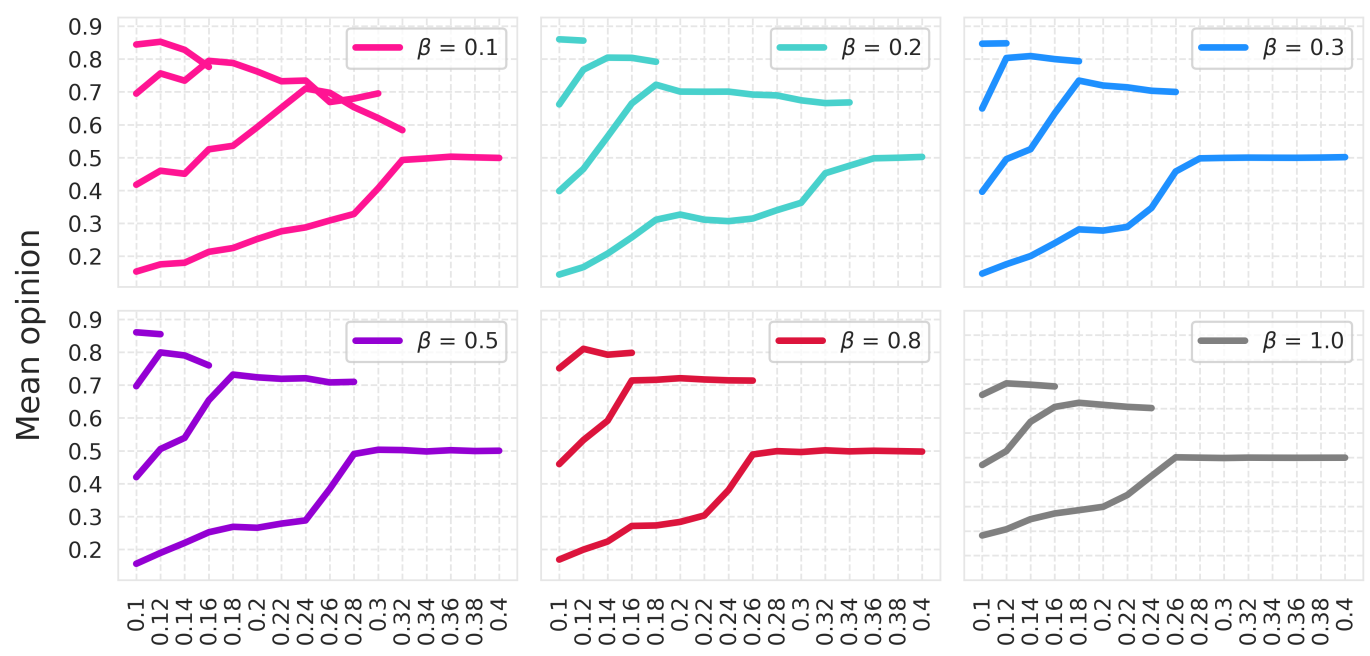

Confidence bound $(C)$

FIG. 5: The means of the opinions of the largest major opinion clusters versus the confidence bound $C$. Each panel gives the results for a different value of the opinion tolerance threshold $\beta$; we show up to four clusters for each value of $\beta$. (We show three major clusters in situations where there are only three of them.) The results that we show are means of the same 50 trials as in Figure 3.

\subsection{Homophilic Rewiring (i.e. $\beta<1$ )}

4.3.1 Shifted Critical Value (i.e. Shifted Phase Transition) When $\beta<1$ (i.e. when there is homophilic rewiring), we often observe that the transition between polarization/fragmentation and consensus occurs at a larger value of $C$ than for the baseline case (i.e. when $\beta=1$ ), especially when $\beta$ is small. That is, consensus is harder to achieve when agents are intolerant of opinions that differ from theirs, as edges are more readily discordant and agents thus rewire more often. In particular, when $\beta$ is small, our model results in a polarized or fragmented steady state for many values of $C$ up to $C_{\beta}^{\prime} \approx 0.34$, which usually results in a consensus steady state for DW models. Additionally, as we see in Figure 4, the transition point $C_{\beta}^{\prime}$ depends on $\beta$ in a complicated way. For example, the trials of our adaptive opinion model with $\beta \approx 0.3$ behave rather differently than those with $\beta \approx 0.2$.

4.3.2 Pseudo-Consensus: Competing Timescales and the Role of Moderate Nodes By allowing nodes to break connections based on their opinion tolerance threshold, we also observe other interesting phenomena. For example, agents can still sometimes reach a consensus - although it is sometimes in the form of a pseudo-consensus - when $C<C_{\beta}^{\prime}$ for certain values of $\beta$. We show an example of a pseudo-consensus in Figures 2(a,b,c). In this example, opinions seemingly converge to one value near the center of the opinion space (see Figure 2(b)). However, upon closer inspection (see Figure 2(c)), we observe subclusters within the steady-state opinion cluster that differ from each other by a small value $\varepsilon \approx 0.0003$. The visualization in Figure 2(a) also suggests that there is some community structure.

It seems that a pseudo-consensus can arise from the presence of two competing processes (rewiring 
and opinion changes) with comparable rates. When there is rewiring, the network tends to form homophilic communities (including situations in which the network itself fragments into multiple components), which one can interpret as echo chambers. Meanwhile, the opinion updates for $C \gtrsim 0.24$ tend to encourage consensus formation because agents are more open-minded and more opinion compromises can occur. If a network organizes into communities faster than agents can come to a consensus, we expect to observe polarization or fragmentation. However, if the agents in a network reach a consensus faster than the network rewires into poorly connected or disconnected communities, we expect the system to reach consensus. When the processes have comparable rates, a pseudo consensus may arise. In such a situation, there is only one opinion cluster, but there appears to be community structure in the cluster (see Figure 2(a)).

To see our reasoning, let's consider the case in which the homophilic rewiring is faster than the opinion changes. Specifically, suppose that both $\beta$ and $C$ are small. When $C$ is small (e.g. $C<0.2$ ), nodes tend to start with fewer neighbors that can influence them than for larger values of $C$, so their opinions change much more slowly at the beginning of a simulation. Calculating the total number of 'influential neighbors' (i.e., the number of neighbors whose opinion is within $C$ of a node) of each node versus time confirms this observation. If $\beta$ is also small (e.g. $\beta<0.2$ ), nodes can very quickly unfriend neighbors whose opinions are too far away from theirs and befriend nodes whose opinion values are closer to theirs. This results in the formation of communities or even multiple connected components in a network. Once the nodes of a network organize into such homophily-based communities, they then compromise their opinions within such communities and quickly achieve an intra-community consensus. (The opinions of the nodes in a community are likely to be within one another's confidence bounds, so two nodes in the same community tend to compromise when they interact.) By this time, there are very few or even no remaining discordant edges, so these communities persist over time. However, when the opinions of a network's nodes converge very quickly (specifically, large $C$ ), the nodes do not have many chances to rewire their connections away from their discordant neighbors to form communities. Therefore, it is reasonable that a pseudo-consensus (i.e. a consensus with subclusters and community structure) emerges when the rates of opinion changes and rewiring are balanced. In Figure 7, we identify the region in the $(\beta, C)$ plane in which we often observe pseudo-consensus.

A small number of nodes in an opinion cluster have opinions that lie somewhere between those in the subclusters that we identified above. Such nodes often have initially moderate opinions (e.g. $x_{i} \approx 0.5 \pm 0.05$ ). We hypothesize that these initially moderate nodes (1) are crucial for allowing the nodes of a network to come to a pseudo-agreement instead of becoming polarized or fragmented and (2) act like 'bridges' to keep a network connected. (In our model, when we observe a polarized steady state, we often also observe two connected components in the network.) We leave a thorough investigation of the role of moderate nodes to future work.

4.3.3 Minor Clusters: Isolated and Extreme Agents In Figure 3(b), we see that there is a significant difference in the number of minor clusters when there is rewiring (i.e. when $\beta<1$ ) than when there is not (i.e. when $\beta=1$ ). Notably, there are very few minor clusters when $C$ and $\beta$ are both small. When $C$ is small, nodes tend to be influenced by only a few other nodes and are thus likely to form small groups or even become isolated. However, when there is rewiring, nodes are able to replace neighbors with discordant opinions from theirs with similar-minded nodes with whom they can potentially become part of a major cluster.

When the opinion tolerance threshold $\beta$ is very small (e.g. $\beta=0.1$ ), the number of minor clusters at steady state increases with the confidence bound $C$. These minor clusters tend to consist of isolated nodes or of 2-3 nodes. Most of the nodes in these minor clusters have initially extreme opinions (i.e. 
(a)

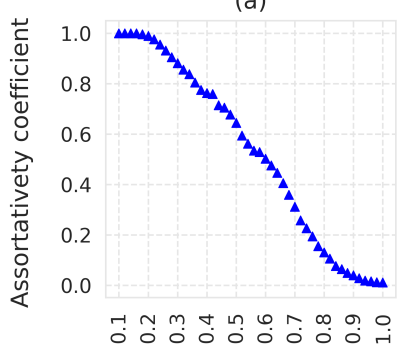

(b)

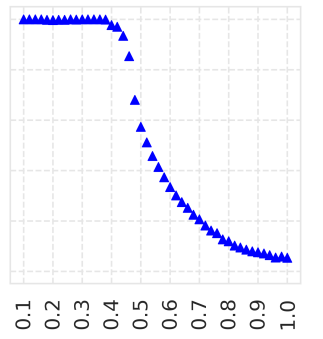

(c)

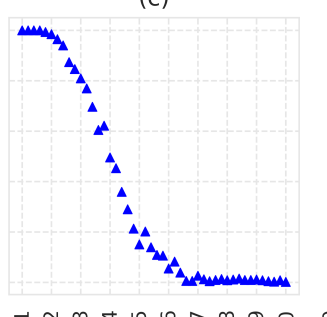

(d)

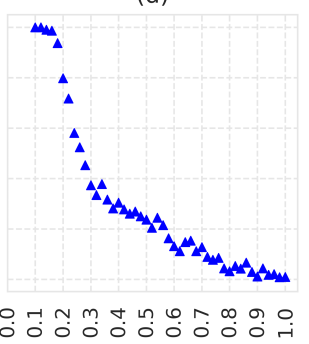

Opinion tolerance threshold $(\beta)$

FIG. 6: Assortativity coefficient $r$ for the final opinion values as a function of the opinion tolerance threshold $\beta$ for (a) $C=0.1$, (b) $C=0.2$, (c) $C=0.3$, and (d) $C=0.4$. The results that we show are means of the same 50 trials as in Figure 3 for values of $\beta$ that we used in that plot.

close to 0 or close to 1 ). The fast formation of a moderate-opinion consensus and the very small value of $\beta$ causes nodes to rewire away from nodes with extreme opinions (i.e. extreme agents) very early in simulations when they become more moderate. The agents that remain extreme are in small clusters at steady state.

The outcomes that we observe in the presence of homophilic rewiring have real-world analogues. For example, for small $\beta$, it is reasonably common in our simulations for small minority groups of extreme agents to be sparsely connected to other parts of a network. These extreme agents cannot be swayed by a majority with more moderate opinions. In the real world, it seems that many people in a population also often disassociate themselves from unpopular minorities. Interestingly, however, the number of such extreme minority groups increases when we increase the amount of open-mindedness of the agents in our model.

4.3.4 Homophily Because we base the rewiring process in our adaptive $\mathrm{BC}$ model on the principle of homophily, it is sensible to compute a measure of homophily in our networks. For simplicity, we calculate the scalar assortativity coefficient [26]

$$
r=\frac{\sum_{i j}\left(A_{i j}-\frac{k_{i} k_{j}}{2 m}\right) x_{i} x_{j}}{\sum_{i j}\left(k_{i} \delta_{i j}-\frac{k_{i} k_{j}}{2 m}\right) x_{i} x_{j}},
$$

where $x_{i}$ is an ordered scalar attribute that is associated with node $i$, the scalar $k_{i}$ is node $i$ 's degree, $m$ is the total number of edges in a network, and $\delta_{i j}$ is the Kronecker delta function. Equation (4.1) is a normalized, network-based generalization of the Pearson correlation efficient. Its value ranges between -1 and 1 , and it attains the value 0 either when there is only one value of the attribute or when there is no tendency for adjacent nodes to be more similar than what one expects in a network in which there is no correlation between the attribute values of adjacent nodes. When $r=1$, the network is perfectly assortative; when $r=-1$, it is perfectly disassortative.

In Figure 6, we show the mean values of the assortativity coefficient $r$ from 50 simulations of our model. In each simulation, $x_{j}$ is the opinion of node $j$ at the end of the simulation and we use the final network structure. These simulations are the same trials as in Figure 3 for values of $\beta$ that we 
(a)
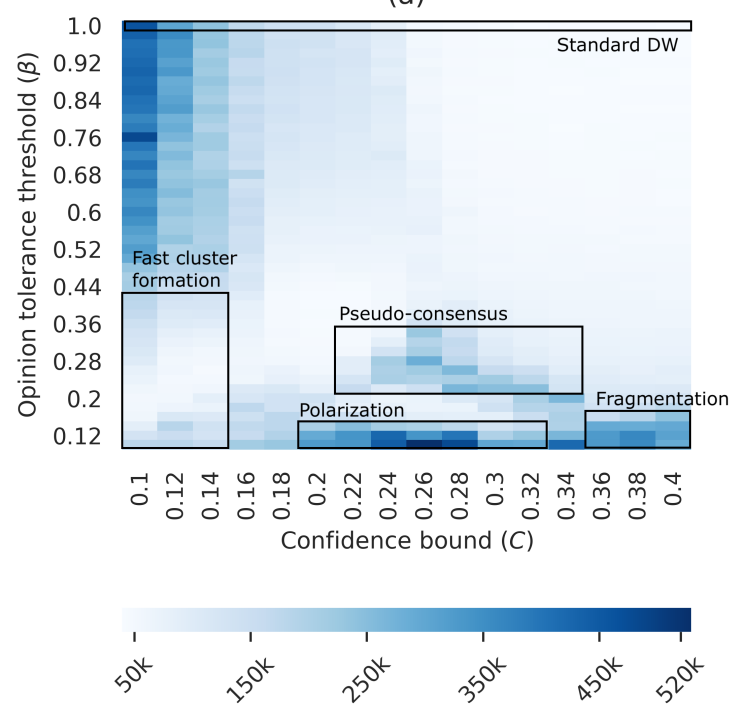

(b)
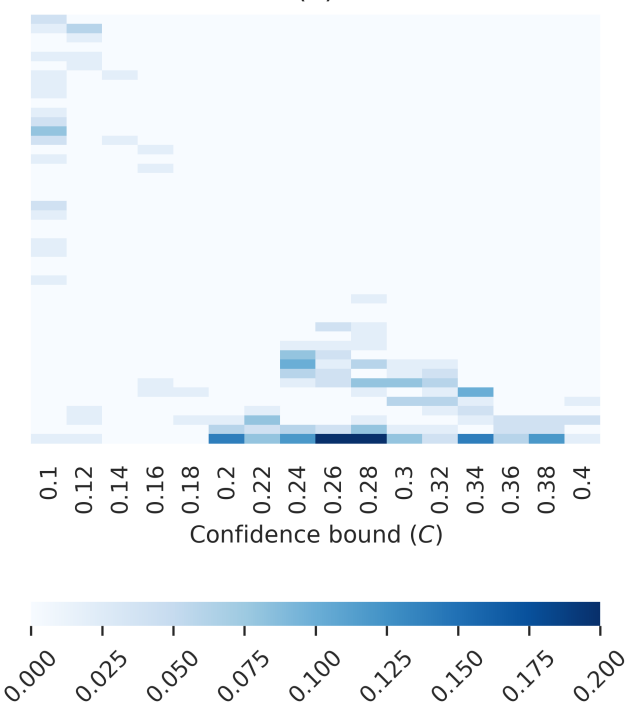

FIG. 7: The (a) convergence time (i.e. the number of steps to reach a steady state) and (b) fraction of trials that reach the bail-out time (i.e. they do not converge within $10^{6}$ steps). The results that we show are means of the same 50 trials as in Figures 3 and 6 for parameter combinations that we showed in those figures. The results for an opinion tolerance threshold of $\beta=1$ correspond to standard DW dynamics (i.e. without rewiring).

considered in that figure. As expected, for any confidence bound $C$, the values of $r$ are large when the opinion tolerance threshold $\beta$ is small. Although $r \approx 1$ (i.e. almost perfect assortative mixing) for all values of $C$ when $\beta$ is very small, different values of $C$ lead to different intervals of $\beta$ for which $r \approx 1$. For example, when $C \in[0.18,0.24]$, the final opinion assortativity $r$ is very large for a large interval of $\beta$ values; the interval of such values is smaller when $C \geqslant 0.3$.

\subsection{Convergence Time}

We roughly organize the $(\beta, C)$ parameter plane into the different steady-state regimes of our model by examining the convergence times of our simulations. In Figure 7(a), we show a heat map that illustrates that the convergence times for intermediate values of $C$ are noticeably slower for small $\beta$ than in the baseline case $\beta=1$ (i.e. when there is no rewiring). We label the associated region in the heat map with the term 'Polarization'. In this region, our simulations reach the bail-out time more frequently than other in regions (see Figure 7(b)).

For small values of the confidence bound $C$, the convergence times of our model are much faster when there is a lot of rewiring (i.e. for small $\beta$ ) than when there is not. See the bottom-left region of the heat map in Figure 7(a). As we discussed previously, in this regime, once the agents in a network organize into homophilic communities through rewiring, they compromise their opinions to achieve intra-community consensus. The pseudo-consensus region also has noticeably different convergence- 
time behavior than the baseline. For a fixed value of $C$, it often takes longer to converge in the former than in the latter. When agents can be influenced both by their own community and by another community, their opinions fluctuate before they come to a moderate-opinion consensus through compromise. When two opinion clusters move closer to forming a pseudo-consensus, opinions can keep changing by minuscule amounts before converging.

\section{Conclusions and Discussion}

We developed an adaptive bounded-confidence model on networks that generalizes the Deffuant-Weisbuch model by incorporating rewiring of discordant edges based on opinion homophily. We studied our model on $G(N, p)$ Erdôs-Rényi networks, and we found that it is harder for networks to achieve consensus when there is rewiring than when there is no rewiring (and hence in the standard DW model). We also observed 'pseudo-consensus' steady states with two subclusters within a consensus opinion group that deviate from each other by a minuscule opinion value. We observed that the convergence times of numerical simulations of our model tend to be long near critical values $C_{\beta}^{\prime}$ of the confidence bound $C$ that separate consensus steady states from polarized and fragmented steady states. We also saw that the convergence times tend to be short when both $C$ and the opinion tolerance threshold $\beta$ are small, in contrast to the typical behavior of the standard DW model. We demonstrated that the number of major clusters at steady state is similar both with rewiring and without rewiring and that large values of $C$ can encourage the formation of minor clusters, whose nodes tend to have extreme opinion values at the beginning of our simulations.

In the present paper, we focused primarily on situations in which opinions change faster than the nodes rewire (i.e. $M<K$ ). Our preliminary investigation of situations with rewiring rates that are faster than opinion dynamics (i.e. $M>K$ ) reveals scenarios in which it is even harder for agents to reach consensus even with very large confidence bounds (such as $C=0.4$ ). It is also worthwhile to further explore the influence of moderate agents on opinion dynamics.

As with other models of opinion dynamics, our adaptive BC model includes various unrealistic assumptions, which we employed for simplicity. For example, we assumed that all agents have the same confidence bound $C$ and the same opinion tolerance threshold $\beta$, and it is more realistic to incorporate heterogeneity in these parameters. Additionally, in our model, each agent who breaks a connection needs to form a new edge, but people can unfriend someone on a social network (e.g. during hotly contested political elections) without forming a connection with someone else. Moreover, in our model, we did not constrain the number of times that an individual can rewire or include a core group of individuals that they will never unfriend. In reality, it is possible that some people will always remain connected to certain other people (e.g. family members or particularly close friends). Relaxing these and other assumptions of our adaptive BC model are worth exploring to help improve understanding of the spread of opinions in social networks in realistic situations.

\section{Acknowledgements}

We thank Heather Zinn Brooks and the other participants of UCLA's Networks Journal Club for helpful comments. We acknowledge financial support from the National Science Foundation (grant number 1922952) through the Algorithms for Threat Detection (ATD) program. 


\section{REFERENCES}

1. Aiello, L. M., Barrat, A., Schifanella, R., Cattuto, C., Markines, B. \& Menczer, F. (2012) Friendship prediction and homophily in social media. ACM Trans. Web, 6(2), 9:1-9:33.

2. Brede, M. (2019) How does active participation affect consensus: Adaptive network model of opinion dynamics and influence maximizing rewiring. Complexity, page 1486909.

3. Brooks, H. Z. \& Porter, M. A. (2020) A model for the influence of media on the ideology of content in online social networks. Phys. Rev. Research, 2, 023041.

4. Bullo, F. (2021) Lectures on Network Systems. Kindle Direct Publishing, 1.5 edition. Available at http://motion.me.ucsb.edu/book-lns/.

5. Castellano, C., Fortunato, S. \& Loreto, V. (2009) Statistical physics of social dynamics. Rev. Mod. Phys., 81, 591-646.

6. Deffuant, G., Neau, D., Amblard, F. \& Weisbuch, G. (2000) Mixing beliefs among interacting agents. Adv. Complex Sys., 03(01n04), 87-98.

7. Durrett, R. T., Gleeson, J. P., Lloyd, A. L., Mucha, P. J., Shi, F., Sivakoff, D., Socolar, J. E. S. \& Varghese, C. (2012) Graph fission in an evolving voter model. Proc. Natl. Acad. Sci. U.S.A., 109(10), 3682-3687.

8. Flaxman, S., Goel, S. \& Rao, J. M. (2016) Filter bubbles, echo chambers, and online news consumption. Public. Opin. Quart., 80, 298-320.

9. Gross, T. \& Blasius, B. (2007) Adaptive coevolutionary networks: A review. J. Royal Soc. Inter, 5(20), 259271.

10. Hegselmann, R. \& Krause, U. (2000) Opinion dynamics and bounded confidence: Models, analysis, and simulation. J. Artif. Soc. Soc. Simul., 5, 3.

11. Hickok, A., Kureh, Y., Brooks, H. Z., Feng, M. \& Porter, M. A. (2021) A bounded-confidence model of opinion dynamics on hypergraphs. SIAM J. App. Dyn. Sys., in press (arXiv:2102.06825).

12. Holley, R. A. \& Liggett, T. M. (1975) Ergodic theorems for weakly interacting infinite systems and the voter model. Ann. Probab., 3(4), 643-663.

13. Khanam, K. Z., Srivastava, G. \& Mago, V. (2020) The homophily principle in social network analysis. arXiv:2008.10383.

14. Kossinets, G. \& Watts, D. J. (2009) Origins of homophily in an evolving social network. Amer. J. Sociol., 2 , https://doi.org/10.1086/599247.

15. Kozma, B. \& Barrat, A. (2008a) Consensus formation on adaptive networks. Phys. Rev. E, 77(1), 016102.

16. Kozma, B. \& Barrat, A. (2008b) Consensus formation on coevolving networks: Groups' formation and structure. J. Phys. A. Math. Theor., 41(22), 224020.

17. Lehmann, S. \& Ahn, Y.-Y. (2018) Complex Spreading Phenomena in Social Systems: Influence and Contagion in Real-World Social Networks. Springer International Publishing, Cham, Switzerland.

18. Li, X., Mobilia, M., Rucklidge, A. M. \& Zia, R. (2021a) Effects of homophily and heterophily on preferreddegree networks: Mean-field analysis and overwhelming transition. arXiv:2107.13945.

19. Li, X., Mobilia, M., Rucklidge, A. M. \& Zia, R. K. P. (2021b) How does homophily shape the topology of a dynamic network?. Phys. Rev. E, 104, 044311.

20. Lorenz, J. (2007) Continuous opinion dynamics under bounded confidence: A survey. Int. J. Mod. Phys. C, 18(12), 1819-1838.

21. Lorenz, J. (2010) Heterogeneous bounds of confidence: Meet, discuss and find consensus!. Complexity, 15(4), 43-52.

22. Malik, N., Shi, F., Lee, H. W. \& Mucha, P. J. (2016) Transitivity reinforcement in the coevolving voter model. Chaos, 26(12), 123112.

23. McPherson, M., Smith-Lovin, L. \& Cook, J. M. (2001) Birds of a feather: Homophily in social networks. Ann. Rev. Sociol., 27(1), 415-444.

24. Mei, W., Bullo, F., Chen, G., Hendrickx, J. M. \& Dorfler, F. (2020) Rethinking the micro-foundation of opinion dynamics: Rich consequences of an inconspicuous change. In 3rd IFAC Conference on Cyber-Physical \& Human-Systems, Shanghai, China. 
25. Meng, X. F., Van Gorder, R. A. \& Porter, M. A. (2018) Opinion formation and distribution in a boundedconfidence model on various networks. Phys. Rev. E, 97(2), 022312.

26. Newman, M. E. J. (2018) Networks. Oxford University Press, Oxford, UK, second edition.

27. Noorazar, H., Vixie, K. R., Talebanpour, A. \& Hu, Y. (2020) From classical to modern opinion dynamics. Int. J. Mod. Phys. C, 31(7), 2050101.

28. Ortiz, E. \& Serrano, M. A. (2021) Multiscale opinion dynamics on real networks. arXiv:2107.06656.

29. Parravano, A., Andina-Díaz, A. \& Meléndez-Jiménez, M. A. (2016) Bounded confidence under preferential flip: A coupled dynamics of structural balance and opinions. PLOS ONE, 11(10), e0164323.

30. Porter, M. A. \& Gleeson, J. P. (2016) Dynamical Systems on Networks: A Tutorial, volume 4. Springer International Publishing, Cham, Switzerland.

31. Sasahara, K., Chen, W., Peng, H., Ciampaglia, G. L., Flammini, A. \& Menczer, F. (2021) Social influence and unfollowing accelerate the emergence of echo chambers. J. Comp. Social Sci., 4, 381-402.

32. Sayama, H., Pestov, I., Schmidt, J., Bush, B. J., Wong, C., Yamanoi, J. \& Gross, T. (2013) Modeling complex systems with adaptive networks. Comp. Math. App., 65(10), 1645-1664.

33. Shi, F., Mucha, P. J. \& Durrett, R. T. (2013) Multiopinion coevolving voter model with infinitely many phase transitions. Phys. Rev. E, 88(6), 062818.

34. Weisbuch, G., Deffuant, G., Amblard, F. \& Nadal, J.-P. (2002) Meet, Discuss, and Segregate!. Complexity, 7(3), 55-63. 